\title{
Nodular lymphocyte-predominant Hodgkin lymphoma (NLPHL) with CD30-positive lymphocyte-predominant (LP) cells
}

\author{
Rania M. Seliem • Judith A. Ferry • \\ Robert P. Hasserjian • Nancy L. Harris • \\ Lawrence R. Zukerberg
}

Received: 28 April 2011 / Accepted: 8 July 2011 / Published online: 23 July 2011

(C) Springer-Verlag 2011

\begin{abstract}
Nodular lymphocyte-predominant Hodgkin lymphoma (NLPHL) is a distinct neoplasm within the spectrum of Hodgkin lymphomas with characteristic clinical, morphological, and immunohistochemical features. According to the WHO definition, lymph nodes involved by NLPHL should have a nodular or nodular and diffuse proliferation of scattered large neoplastic lymphocyte-predominant (LP) cells in a small cell background that reside in expanded follicular dendritic cell meshworks; the LP cells must also have a distinct immunophenotypic profile. The LP cells are monoclonal B cells that are typically CD20, BCL6, CD79a, and CD45 positive and are CD30 and CD15 negative. In contrast, the Reed-Sternberg cells of classical Hodgkin lymphoma (CHL) are typically positive for $\mathrm{CD} 15$ and CD30. However, in CHL, CD20 staining is variable, and CD15 staining may be absent in some cases. Thus, CD30 is often considered to be the most distinctive marker between CHL and NLPHL. In order to better assess CD30 staining in NLPHL, we reviewed 220 cases of NLPHL and found 21 cases that showed at least focal staining of the neoplastic cells for CD30. The CD30 staining was often faint, but occasionally strong, and typically was found only on a subset of the LP cells. We evaluated the clinicopathologic features of these cases to determine whether they showed differences from typical CD30-negative NLPHL and found no significant difference with respect to clinical presentation, histology, other immunophenotypic features or out-
\end{abstract}

R. M. Seliem • J. A. Ferry • R. P. Hasserjian • N. L. Harris

L. R. Zukerberg $(\triangle)$

Department of Pathology, Massachusetts General Hospital,

Harvard Medical School,

55 Fruit Street, Warren Building 2nd floor,

Boston, MA 02114, USA

e-mail: lzukerberg@partners.org come. In summary, we conclude that CD30 expression by LP cells in NLPHL can be seen and should not lead to a misdiagnosis of CHL. The presence of CD30-positive LP cells is not associated with other features of CHL or unusually aggressive behavior.

Keywords Nodular lymphocyte-predominant Hodgkin lymphoma $\cdot \mathrm{CD} 30 \cdot \mathrm{LP}$ cells

\section{Introduction}

Hodgkin lymphomas have many important features that separate them from other lymphomas. They most often occur in the lymph nodes of young adults and contain a small number of neoplastic cells intermingled with numerous reactive inflammatory and accessory cells $[1,2]$. Hodgkin lymphomas are separated into two disease entities: nodular lymphocyte-predominant Hodgkin lymphoma (NLPHL) [2] and classical Hodgkin lymphoma (CHL) [1]. These two entities differ in their clinical and pathologic features.

CHL is a neoplastic lymphoid proliferation composed of mononuclear Hodgkin cells and bi- or multinucleated ReedSternberg (RS) cells that proliferate within an inflammatory background composed of small lymphocytes, eosinophils, neutrophils, histiocytes, fibroblasts, and collagen fibers. The neoplastic RS cells are large with bilobed or multilobated nuclei that have vesicular chromatin and prominent eosinophilic nucleoli. RS cells are positive for CD30 in virtually all cases [3], and for CD15 [3-5] in the majority of cases. The RS cells are typically negative for CD45, CD79a, and BCL6. In approximately $40 \%$ of cases, CD20 may be detectable with variable intensity and present only 
on a minority on the neoplastic cells $[6,7]$. B cell-specific transcription factor, a product of the PAX5 gene, is dimly positive in approximately $90 \%$ of the cases [8]. The B cell transcription factors OCT2 and/or its coactivator BOB1 are variably present, and at least one is usually weak or absent in CHL [9]. Epstein-Barr virus (EBV) is present in RS cells in $10-70 \%$ of the cases depending on subtype. CHL makes up $95 \%$ of Hodgkin lymphomas with a bimodal age distribution in late adolescence/young adulthood and again in the elderly. CHL most often involves lymph nodes of the cervical region followed by the mediastinal, axillary, and para-aortic regions and is curable in most cases with current treatment regimens.

NLPHL is a monoclonal B cell neoplasm with a nodular or at least partially nodular proliferation of neoplastic lymphocyte-predominant (LP) cells within a background of mainly small B lymphocytes and scattered histiocytes, some of which may be epithelioid. The LP cells usually reside within follicular dendritic cell (FDC) meshworks. The LP cells are large with oval, folded, or multilobated nuclei with vesicular chromatin and basophilic nucleoli. They can be indistinguishable from large centroblasts and also from classical HRS cells by cellular morphology alone. The LP cells have a distinct immunophenotypic profile. The cells are positive for CD20 [10-12], BCL6, CD79a, and CD45 in nearly all the cases $[13,14]$. LP cells lack CD15 and CD30 and are negative for EBV. The cells are positive for both OCT2 and BOB1, with characteristically intense staining for OCT2 [9]. Their immunophenotype thus closely approximates that of germinal center B cells. NLPHL patients are mostly young males ranging from 30 50 years, and the main sites of involvement are cervical, axillary, or inguinal lymph nodes. Mediastinal, spleen, and bone marrow involvement is rare [2]. NLPHL is relatively infrequent (comprising approximately $5 \%$ of all Hodgkin lymphoma), and has a relatively good prognosis with a 10year survival of more than $80 \%$ [15]. Late recurrences may occur, however, and some patients may relapse as diffuse large B cell lymphoma.

In most cases, distinction between NLPHL and CHL is straightforward by morphology. However, this distinction may be difficult in some cases, especially those of the lymphocyte-rich subtype. Lymphocyte-rich classical Hodgkin lymphoma (LRCHL) was delineated as a specific entity within CHL in the 2008 WHO Classification. In LRCHL, the RS cells have the typical immunophenotype described in CHL. However, these cells reside in a background rich in lymphocytes and often occur in association with small B cell follicles. LRCHL thus bears some histologic similarities to NLPHL; indeed, prior to the widespread use of paraffin section immunostaining in lymphoma diagnosis, many LRCHL were misclassified as NLPHL [16]. Distinction of CHL from NLPHL should be possible by immunophenotyping, in cases which are not morphologically distinct. However, the neoplastic cells in CHL can express CD20 variably, and CD15 staining can be absent in some cases. CD30 would therefore seem to be the most distinctive marker between CHL and NLPHL. In order to better assess if LP cells can occasionally express CD30, we reviewed 220 cases of NLPHL and found 21 cases that showed at least focal staining of the LP cells for CD30. A detailed analysis of these 21 cases is reported to ascertain if they have distinct clinicopathologic features that differ from typical NLPHL.

\section{Materials and methods}

We retrospectively analyzed the morphologic and immunohistochemical features of 21 cases of NLPHL that showed at least focal staining of LP cells for CD30, from 220 cases of NLPHL evaluated in our institution from 1998 to September 2005. CD30-positive cases were chosen by reviewing the pathology reports of the 220 NLPHL cases that had been stained for $\mathrm{CD} 30$, and any case that mentioned CD30 positivity or questionable CD30 staining (i.e., was not called negative) was reviewed. Cases in which $\mathrm{CD} 30+$ cells were present but did not correspond to LP cells were not included in the study. In particular, immunoblasts, which are often $\mathrm{CD} 30+$, were distinguished from LP cells. Ninety percent of the cases were submitted in consultation to one of the authors (N.L.H or J.A.F.). The morphologic features of NLPHL were evaluated using H\&E-stained slides.

Immunohistochemical analysis was performed on $2 \mu \mathrm{m}$ thick formalin fixed, paraffin embedded sections using Ventana 3, 3' diaminobenzidine tetrahydochloride kit according to the manufacturer's instructions. Antibodies were tested against CD30 (Ventana, Tucson, AZ), CD15 (Ventana), CD45 (DAKO, Carpinteria, CA), BOB-1 (Santa Cruz, Santa Cruz, CA), CD20 (Dako), OCT-2 (Santa Cruz), BCL-6 (DAKO), CD21 (Ventana), Pax-5 (Ventana), and in situ hybridization for EBV (Ventana) was also evaluated. Appropriate positive controls were included with all analyses. Staining for CD30 was graded semiquantitatively as 0 (no staining), $1+$ (faint staining), or $2+$ (strong staining). The relative number of CD30-positive LP cells was graded as rare $(<5 \%$ of all LP cells), few $(5-20 \%)$, or many $(>20 \%)$.

Detailed clinicopathologic features were analyzed in the 21 cases that showed at least focal faint or strong staining of LP cells for CD30. Clinical information was available in all cases from the pathology report and accompanying referring letter or the electronic medical record. Clinical information recorded included patient age, gender, sites of involvement, treatment (when available), and outcome at 
latest available follow-up. Institutional review board approval was obtained from Partners Healthcare System.

\section{Results}

The clinical features are summarized in Table 1. Eighteen patients were men and three were women, with a median age of 49 years and a mean age of 43 years (range 5 to 71 years). All but two patients had solitary enlarged lymph node groups involving inguinal/femoral lymph nodes (eight cases), cervical lymph nodes (five cases), axillary lymph nodes (four cases), and mesenteric lymph nodes (two cases). One patient had both hilar and retroperitoneal lymphadenopathy, and one patient had a nasopharyngeal mass. Clinical follow-up was available for 17 of the 21 patients. The follow-up period ranged from 4 months to 18 years (median, 5 years). Detailed treatment plans were available in five cases and included a "watch and wait" approach, localized radiation, and systemic chemotherapy.
Thirteen of 17 patients did not show any evidence of disease recurrence or progression. One patient (case 5) had recurrent disease in the form of diffuse large $B$ cell lymphoma and died of acute respiratory distress 11 months later. One patient (case 17) had recurrence of NLPHL in a distant site 7 years after the initial lesion and is alive with no evidence of disease (ANED) 4 years after the recurrence. Similarly, one patient (case 19) had recurrence of NLPHL 7 years after initial presentation and is ANED 3 years after the recurrence. The fourth patient (case 21) had a recurrence 11 years after the initial diagnosis. All cases showed typical morphologic features of NLPHL with an entirely or partially nodular proliferation of small lymphocytes, histiocytes, and intermingled LP cells (Figs. 1a, b, 2a). Epithelioid histocytes were present in 11 of the 21 cases and focally ringed the nodules. Eight of the 21 cases showed variable degrees of sclerosis and fibrous band formation (38\%; Fig. 1a). In most cases, this was limited to a small amount of fibrosis at the periphery of some of the nodules. However, in four cases, the sclerosis was promi-

Table 1 Clinical features of the patients

\begin{tabular}{|c|c|c|c|c|c|}
\hline Case & $\begin{array}{l}\text { Age } \\
\text { (years) }\end{array}$ & Sex & Presentation & $\begin{array}{l}\text { Follow-up } \\
\text { interval }\end{array}$ & Clinical follow-up \\
\hline 1 & 58 & $\mathrm{~F}$ & Right inguinal lymphadenopathy & 5 years & Negative clinical \\
\hline 2 & 9 & $\mathrm{~F}$ & Right cervical lymphadenopathy & 5 years & NA \\
\hline 3 & 21 & M & Left inguinal lymphadenopathy & $51 / 2$ years & Negative clinical and radiological examination \\
\hline 4 & 12 & M & Right cervical lymphadenopathy & 5.5 years & Negative clinical and radiological examination \\
\hline 5 & 71 & M & $\begin{array}{l}\text { Hilar and retroperitoneal } \\
\text { lymphadenopathy }\end{array}$ & 18 years & $\begin{array}{l}\text { Large cell lymphoma with relapse and NLPHD; died } \\
\text { with ARDS }\end{array}$ \\
\hline 6 & 25 & M & Left leg lymphadenopathy & 6.5 years & Negative clinical and radiological examination \\
\hline 7 & 56 & M & Mesenteric lymphadenopathy & 6 years & Negative clinical examination \\
\hline 8 & 54 & M & Mesenteric lymphadenopathy & 5 years & Negative clinical and radiological examination \\
\hline 9 & 25 & M & Cervical lymphadenopathy & 3 years & Negative clinical examination \\
\hline 10 & 67 & M & Left inguinal lymphadenopathy & 3 years & Negative clinical examination \\
\hline 11 & 38 & M & Cervical lymphadenopathy & 3.5 years & NA \\
\hline 12 & 57 & M & Nasopharyngeal mass & 3.5 years & Negative BM biopsy and clinical examination \\
\hline 13 & 55 & M & Right inguinal lymphadenopathy & 2.5 years & Negative LN biopsy and CT \\
\hline 14 & 57 & M & Right axillary lymphadenopathy & 1.5 years & Negative CT \\
\hline 15 & 58 & M & Left inguinal lymphadenopathy & 1.5 years & Negative CT and clinical examination \\
\hline 16 & 56 & M & Left axillary lymphadenopathy & 4 months & NA \\
\hline 17 & 39 & M & Left femoral lymphadenopathy ${ }^{\mathrm{b}}$ & 10 years & Negative CT and LN biopsy \\
\hline 18 & 49 & $\mathrm{~F}$ & Left femoral lymphadenopathy & 4 years & Negative $\mathrm{CT}$ and clinical examination \\
\hline 19 & 39 & M & Right cervical lymphadenopathy ${ }^{\mathrm{c}}$ & 10 years & Negative clinical examination \\
\hline 20 & 5 & M & Left arm lymphadenopathy & 2 years & NA \\
\hline 21 & 42 & M & Right axillary lymphadenopathy & 11 years & Recurrence 11 years later \\
\hline
\end{tabular}

${ }^{a}$ DLBCL 18 years earlier; NLPHL 7 years earlier with no CD30+ cells and then relapse of DLBCL and NLPHL with CD30+ cells; died 11 months later with ARDS

${ }^{\mathrm{b}}$ Initial presentation in left axilla treated with radiation alone with groin recurrence 7 years later

${ }^{\mathrm{c}}$ Initial presentation in left axilla 7 years earlier that was CD30 negative, treated with radiation alone, negative clinical examination 3 years after recurrence 

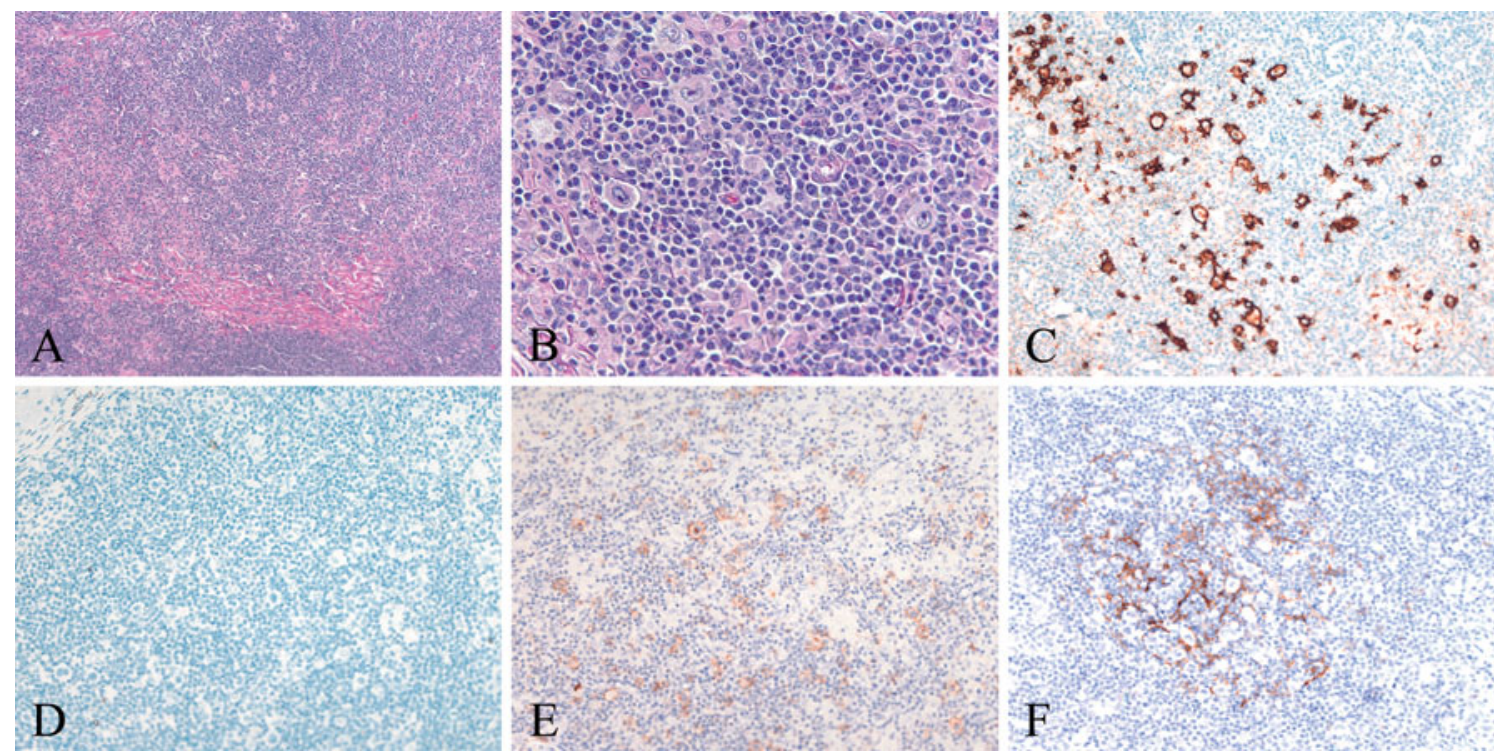

Fig. 1 NLPHL with focal sclerosis and many CD30+ LP cells (case 20). a Low power examination shows focal somewhat irregular, bandlike fibrosis at the periphery of an ill-defined nodule. b High power shows a predominance of small lymphocytes with few scattered histiocytes and scattered large atypical LP cells with large, pale, oval, indented, or lobated nuclei and distinct nucleoli. c An immunostain for CD20 shows strong staining of LP cells. Only a minority of the small

nent with broad bands of sclerosis and extensive hyalinetype sclerosis. All cases showed varying numbers of immunoblasts that were more numerous between the nodules, but some were scattered within the neoplastic nodules.

Immunostains were performed on all cases and are summarized in Table 2. In all 21 cases, the LP cells were lymphocytes are $\mathrm{CD} 20+$, in contrast to the usual predominance of $\mathrm{B}$ cells among the small lymphocytes in NLPHL. $\mathbf{d}$ There is no staining for CD15. e With antibody to CD30, there is bright (2+) staining of many LP cells. f CD21 highlights an expanded but somewhat attenuated follicular dendritic meshwork associated with a cellular nodule within the NLPHL (c-f immunoperoxidase technique on paraffin sections)

strongly and uniformly CD20 positive and CD15 negative (Fig. 1c, d). In all cases studied, the LP cells were positive for CD45 (8/8 cases), Pax5 (6/6 cases), BCL6 (8/8 cases), and for both OCT2 and BOB1 (8/8 cases), and were negative for EBV-encoded small nuclear RNA (EBER; 0/9 cases; Fig. 2b). As defined by our criteria, all 21 cases had some CD30-positive LP cells. In most cases (14/21 cases,
Fig. 2 NLPHL with rare CD30 + LP cells (case 19). a High power shows large, pale LP cells in a background of small lymphocytes. b LP cells and scattered small B cells show strong nuclear staining for OCT2. c. With antibody to CD30, there are a few faintly positive LP cells (arrows). Also seen are few scattered immunoblasts expressing CD30 more strongly than the LP cells. $\mathbf{d}$ The LP cells are present within an irregularly expanded CD21+ follicular dendritic meshwork (b-d immunoperoxidase technique on paraffin sections)
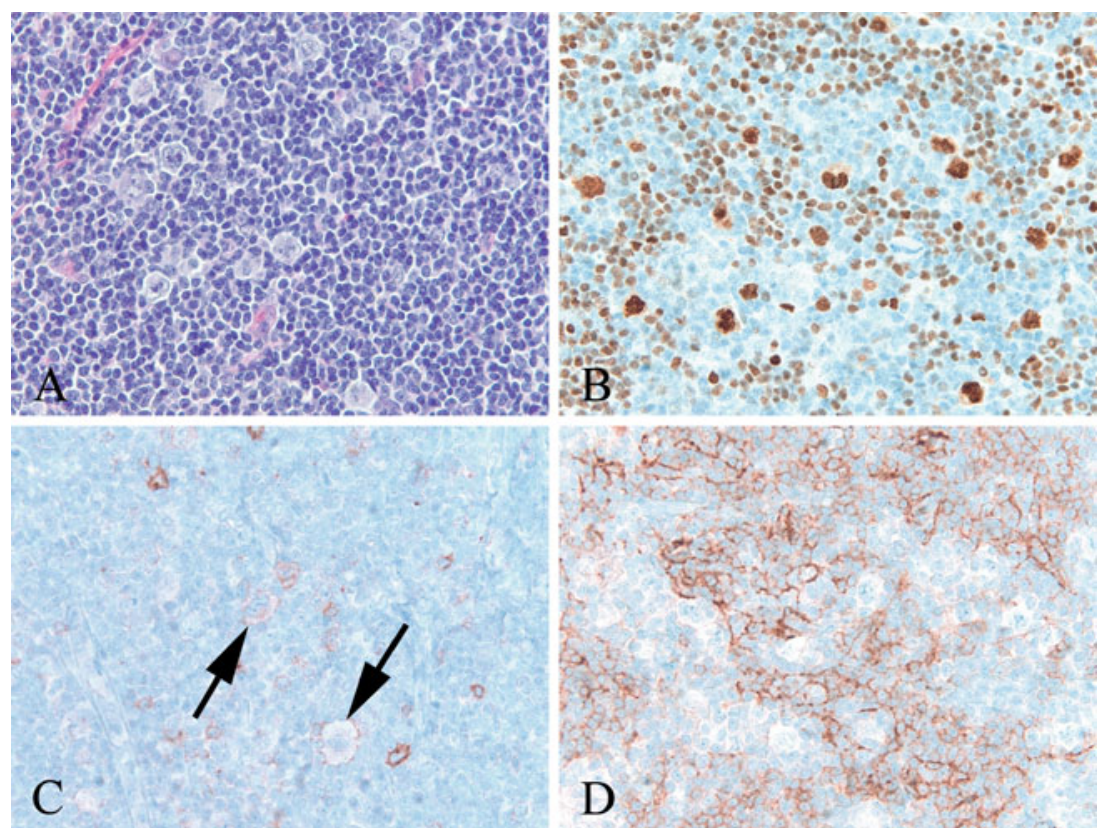
Table 2 The immunostains performed on all cases

+ faint staining of LP cells, ++ strong staining of LP cells

$N D$ not done

\begin{tabular}{|c|c|c|c|c|c|c|c|}
\hline Case & CD30 staining in LP cells & $\mathrm{CD} 45$ & $\mathrm{CD} 20$ & PAX5 & BCL6 & BOB1/OCT2 & EBER \\
\hline 1 & + , rare & + & + & ND & ND & ND & ND \\
\hline 2 & ++ , rare & ND & + & ND & ND & ND & - \\
\hline 3 & ++ , rare & $\mathrm{ND}$ & + & ND & ND & ND & ND \\
\hline 4 & ++ , rare & + & + & ND & ND & ND & ND \\
\hline 5 & ++ , rare & ND & + & ND & ND & ND & - \\
\hline 6 &,+ rare & ND & + & + & + & ND & - \\
\hline 7 &,+ rare & + & + & ND & ND & ND & ND \\
\hline 8 & + , rare & ND & + & ND & + & ND & ND \\
\hline 9 & + , rare & ND & + & + & + & $+/+$ & ND \\
\hline 10 & ++ , many & + & + & ND & + & $+/+$ & - \\
\hline 11 & + , rare & + & + & ND & ND & ND & ND \\
\hline 12 & ++ , many & ND & + & + & + & $+/+$ & - \\
\hline 13 & + , rare & ND & + & ND & ND & ND & ND \\
\hline 14 & + , rare & + & + & ND & ND & ND & - \\
\hline 15 & + , rare & Faint + & + & ND & ND & $+/+$ & - \\
\hline 16 & + , rare & ND & + & ND & ND & $+/+$ & ND \\
\hline 17 & + , rare & ND & + & ND & ND & ND & ND \\
\hline 18 & + , rare & ND & + & ND & ND & ND & ND \\
\hline 19 &,+ rare & $\mathrm{ND}$ & + & + & + & $+/+$ & - \\
\hline 20 & ++ ,many & + & + & + & + & $+/+$ & - \\
\hline 21 & + , rare & ND & + & + & + & $+/+$ & ND \\
\hline
\end{tabular}

cytometric analysis frequently discloses a subset of $\mathrm{T}$ cells with co-expression of CD4 and CD8 (double positive T cells) without markers of an immature stage of differentiation including $\mathrm{TdT}$ and $\mathrm{CD} 1 \mathrm{a}$; this likely represents an unusual reactive or activated $\mathrm{T}$ cell population [18]. The differential diagnosis of NLPHL is broad. As LP cells are typically present in small numbers, they may be overlooked, and NLPHL may be misinterpreted as a reactive process. The nodular pattern may suggest follicular lymphoma. If $\mathrm{T}$ cells are abundant and the CD4/CD8 ratio is high, the possibility of peripheral $\mathrm{T}$ cell lymphoma could be considered. T cells with co-expression of CD4 and CD8 may raise the question of T-lymphoblastic lymphoma. Rare cases of NLPHL with morphologically atypical T cells can also be mistaken for peripheral $\mathrm{T}$ cell lymphoma [19]. Misdiagnosis can be avoided if the pathologist is familiar with the range of morphologic and immunophenotypic features that may be seen in cases of NLPHL.

Occasional cases of NLPHL show CD30 expression by the LP cells, and this finding may raise the question of classical Hodgkin lymphoma (CHL) [20], especially lymphocyte-rich CHL, but because of the presence of fibrosis around some nodules, the differential may also include nodular sclerosis CHL. Thus, we evaluated cases of NLPHL for CD30 expression to see how frequently it occurred and if the positive cases had different clinicopathologic features from typical NLPHL. Approximately $10 \%$ $(21 / 220)$ cases of NLPHL in our series showed at least 
some positive staining for CD30 in LP cells. However, only three $(1 \%)$ cases showed strong CD30 expression in many cells, while another four cases $(2 \%)$ showed strong staining in scattered cells. This is in contrast to CHL in which ReedSternberg cells and variants all typically strongly express CD30.

CD30 expression by LP cells has been described previously in smaller series, although frequencies both higher and lower than found in this study have been described. One study of NLPHL showed that 7\% (4/59) of cases showed CD30 expression [21]. Another study showed $19 \%$ of cases with focal LP cell staining for CD30; this relatively high frequency was attributed to heat epitope retrieval artifact [17]. However, others have not found CD30 expression in NLPHL, even using enhanced antigen retrieval [22]; this might be due to a small number of cases in that series as only 16 cases were studied. As most of the cases in our series were referred from other pathologists, there may have been some degree of referral bias selecting for cases that showed CD30 expression.

The most substantial challenge in many cases is distinguishing CD30+ immunoblasts from LP cells. Many of the cases submitted in consultation with a question of CD30 expression by LP cells in fact showed CD30 expression by reactive immunoblasts. We took great care to distinguish LP cells from other cells, such as immunoblasts, and if a cell was questionable it was considered an immunoblast. These may be more numerous than the LP cells and in general show stronger CD30 staining. CD30positive immunoblasts were present both outside and within the nodules, although in most cases they were more prominent around the nodules. Most of our positive cases showed weaker CD30 staining of the LP cells than the immunoblasts, but in seven cases, LP cells showed equal or stronger staining. However, most of the latter (4/7 cases) had only rare scattered positive LP cells.

The 21 cases in our series had typical morphology of NLPHL, except for more frequent sclerosis or fibrous bands than has been described. Almost $40 \%$ of the cases showed some sclerosis or fibrous bands, but only in four cases (\#2, \#5, \#10, \#19) was the sclerosis prominent. Prominent sclerosis was reported by Fan et al. in $22 \%$ of cases and in their study of variant patterns of NLPHL and was associated with recurrent disease [17]. In our experience, we find sclerosis not infrequently in otherwise typical cases of NLPHL and do not believe it is particular to CD30positive NLPHL, and certainly does not exclude a diagnosis of NLPHL. CD30-positive lymphoproliferative disorders have been reported to be associated with c-Jun expression and activation, which is an activator of the activator protein-1 (AP1) transcription factor pathway [23]. AP1 acts as a transcription factor for transforming growth factorbeta which is known to induce fibrosis. Thus, it is possible that the sclerosis seen in the CD30-positive cases may indicate an activated c-Jun pathway. It would be interesting to determine whether the CD30-positive NLPHL cases have activated c-Jun similar to CHL as cases of NLPHL were reported as negative for this feature in a prior study [23].

Other than the CD30 staining, the immunophenotype of our cases was identical to that typically reported for NLPHL with staining for CD45 and B cell markers and lacking CD15 expression and EBER. Thus, in questionable cases that may show strong CD30 expression by many LP cells (three cases in our study), the expression of B cell markers, such as CD79a, OCT2, and BOB1, in addition to CD20 and nodular aggregates of CD21 FDCs should aid in the diagnosis. In addition, PAX5 was strongly positive in the LP cells in all cases studied, while in most cases of CHL, it is weak and/or focal. The 21 cases had also had clinical features similar to typical NLPHL. The patients were predominantly males in the 40 to 50 -year-old age range who presented with localized peripheral lymphadenopathy of the cervical, axillary, and inguinal lymph nodes. The incidence of recurrent/progressive disease in our series (4/17 cases, $23 \%)$ is not significantly different from that reported for typical NLPHL [24, 25].

In summary, we conclude that faint and even sometimes strong CD30 expression by LP cells in NLPHL can be seen in up to $10 \%$ of cases, usually in only a subset of LP cells. In the presence of histologic and immunophenotypic features that are otherwise typical of NLPHL, CD30 expression, particularly if weak or restricted to a minority of LP cells, should not be used to exclude a diagnosis of NLPHL and confirm a diagnosis of CHL. CD30 expression does not appear to be associated with unusual or aggressive behavior, although additional studies of patients with NLPHL would be helpful in confirming this observation.

Conflict of interest statement The authors declare that they have no conflict of interest.

\section{References}

1. Stein $\mathrm{H}$ et al (2008) Classical Hodgkin lymphoma, introduction. In: Swerdlow S, Campo E, Harris N, Jaffe E, Pileri S, Stein H, Thiele J, Vardiman J (eds) WHO classification of tumours of haematopoietic and lymphoid tissues. IARC, Lyon, pp 326329

2. Poppema S et al (2008) Nodular lymphocyte predominant Hodgkin lymphoma. In: Swerdlow S, Campo E, Harris N, Jaffe E, Pileri S, Stein H, Thiele J, Vardiman J (eds) WHO classification of tumours of haematopoietic and lymphoid, 4th edn. IARC, Lyon, pp 323-325

3. Stein $\mathrm{H}$ et al (1985) The expression of the Hodgkin's disease associated antigen $\mathrm{Ki}-1$ in reactive and neoplastic lymphoid tissue: 
evidence that Reed-Sternberg cells and histiocytic malignancies are derived from activated lymphoid cells. Blood 66:848-858

4. Stein H, Uchanska-Ziegler B, Gerdes J, Ziegler A, Wernet P (1982) Hodgkin and Sternberg-Reed cells contain antigens specific to late cells of granulopoiesis. Int J Cancer 29(3):283-290

5. Hsu SM, Jaffe ES (1984) Leu M1 and peanut agglutinin stain the neoplastic cells of Hodgkin's disease. Am J Clin Pathol 82(1):29 32

6. Schmid C, Pan L, Diss T, Isaacson P (1991) Expression of B-cell antigens by Hodgkin's and Reed-Sternberg cells. Am J Pathol 139:701-707

7. Zukerberg L, Collins A, Ferry J, Harris N (1991) Coexpression of CD15 and CD20 by Reed-Sternberg cells in Hodgkin's disease. Am J Pathol 139:475-483

8. Foss H-D et al (1999) Frequent expression of the B-cell-specific activator protein in Reed-Sternberg cells of classical Hodgkin's disease provides further evidence for its B-cell origin. Blood 94 (9):3108-3113

9. Laumen H, Nielsen PJ, Wirth T (2000) The BOB.1/OBF.1 coactivator is essential for octamer-dependent transcription in $\mathrm{B}$ cells. Eur J Immunol 30(2):458-469

10. Pinkus G, Said J (1985) Hodgkin's disease, lymphocyte predominance type, nodular - a distinct entity? Unique staining profile of L\&H variants of Reed-Sternberg cells defined by monoclonal antibodies to leukocyte common antigen, granulocyte specific antigen, and B-cell specific antigen. Am J Pathol 116:1-6

11. Pinkus G, Said J (1988) Hodgkin's disease, lymphocyte predominance type, nodular - further evidence for a B cell derivation: L\&H variants of Reed-Sternberg cells express L26, a pan B cell marker. Am J Pathol 133:211-217

12. Coles F, Cartun R, Pastuszak W (1988) Hodgkin's disease, lymphocyte-predominant type: immunoreactivity with B-cell antibodies. Mod Pathol 1:274-285

13. Poppema S (1980) The diversity of the immunohistological staining pattern of Sternberg-Reed cells. J Histochem Cytochem 28:788-791

14. Stein $\mathrm{H}$ et al (1986) Reed-Sternberg and Hodgkin cells in lymphocyte-predominant Hodgkin's disease of nodular subtype contain J chain. Am J Clin Pathol 86:292-297

15. Diehl V et al (1999) Clinical presentation, course, and prognostic factors in lymphocyte-predominant Hodgkin's disease and lymphocyte-rich classical Hodgkin's disease: report from the
European Task Force on Lymphoma Project on lymphocytepredominant Hodgkin's disease. J Clin Oncol 17:776-783

16. Anagnostopoulos I et al (2000) European Task Force on Lymphoma project on lymphocyte predominance Hodgkin disease: histologic and immunohistologic analysis of submitted cases reveals 2 types of Hodgkin disease with a nodular growth pattern and abundant lymphocytes. Blood 96(5):1889-1899

17. Fan Z, Natkunam Y, Bair E, Tibshirani R, Warnke R (2003) Characterization of variant patterns of nodular lymphocyte predominant Hodgkin lymphoma with immunohistologic and clinical correlation. Am J Surg Pathol 27:1346-1356

18. Rahemtullah A, Reichard KK, Preffer FI, Harris NL, Hasserjian $\mathrm{RP}$ (2006) A double-positive CD4 + CD8+ T-cell population is commonly found in nodular lymphocyte predominant Hodgkin lymphoma. Am J Clin Pathol 126(5):805-814

19. Sohani A, Jaffe E, Harris N, Ferry J, Hasserjian R (2011) Nodular lymphocyte predominant Hodgkin lymphoma with atypical $\mathrm{T}$ cells: a morphologic variant mimicking peripheral T-cell lymphoma. Am J Surg Pathol (in press)

20. Mourad WA et al (2008) Morphologic, immunphenotypic and clinical discriminators between T-cell/histiocyte-rich large B-cell lymphoma and lymphocyte-predominant Hodgkin lymphoma. Hematol Oncol Stem Cell Ther 1(1):22-27

21. Uherova P, Valdez R, Ross CW, Schnitzer B, Finn WG (2003) Nodular lymphocyte predominant Hodgkin lymphoma. An immunophenotypic reappraisal based on a single-institution experience. Am J Clin Pathol 119(2):192-198

22. Roberts C, Jack F, Angus B, Reid A, Thompson WD (2002) Immunohistochemical detection of $\mathrm{CD} 30$ remains negative in nodular lymphocyte-predominant Hodgkin's disease using enhanced antigen retrieval. Histopathology 40(2):166-170

23. Drakos E et al (2007) c-Jun expression and activation are restricted to $\mathrm{CD} 30+$ lymphoproliferative disorders. Am J Surg Pathol 31(3):447-453

24. Yang DT, Dunphy CH, Tripp SR, Lagoo AS, Perkins SL (2008) Nodular lymphocyte predominant Hodgkin lymphoma at atypical locations may be associated with increased numbers of large cells and a diffuse histologic component. Am J Hematol 83(3):218-221

25. Biasoli I et al (2010) Nodular, lymphocyte-predominant Hodgkin lymphoma: a long-term study and analysis of transformation to diffuse large B-cell lymphoma in a cohort of 164 patients from the Adult Lymphoma Study Group. Cancer 116(3):631-639 